Saudi Journal of Oral and Dental Research

Abbreviated Key Title: Saudi J Oral Dent Res

ISSN 2518-1300 (Print) |ISSN 2518-1297 (Online)

Scholars Middle East Publishers, Dubai, United Arab Emirates

Journal homepage: https://saudijournals.com

Review Article

\title{
Back to Basics-Conventional Versus Modern Prosthodontics: A Review
}

Dr. Suchita Sharma ${ }^{1 *}$, Dr. Kapil Sharma ${ }^{2}$

${ }^{1}$ Assistant Professor, Dept. of Prosthodontics and Crown and Bridge and Oral Implantology RDCH, Jaipur Rajasthan India

${ }^{2}$ Assistant Professor, Dept. of Orthodontics and Dentofacial Orthopedics Rajasthan Dental College \& Hospital, Jaipur Rajasthan India

DOI: $10.36348 /$ sjodr.2021.v06i02.001

| Received: 14.01.2021 | Accepted: 25.01.2021 | Published: 03.02.2021

*Corresponding author: Dr. Suchita Sharma

\section{Abstract}

Digitilization has brought about a paradigm shift in the perception and conceptualisation of dentistry, but the conventional concepts and methods deserve due consideration and preservance. After review and comparison of various conventional and digital procedures in prosthodontics it was inferred that conventional methods are almost at par with digital methods except in terms of time constraints and patient comfort.

Keywords: digital technology, conventional,algorithms, CAD CAM, impressions, dental implants, osseodensification.

Copyright $\odot 2021$ The Author(s): This is an open-access article distributed under the terms of the Creative Commons Attribution 4.0 International License (CC BY-NC 4.0) which permits unrestricted use, distribution, and reproduction in any medium for non-commercial use provided the original author and source are credited.

\section{INTRODUCTION}

Broad dissemination of digital technology in dentistry began in the early 1990s with the introduction of digital radiography-computer-assisted design and computer-assisted manufacturing (CAD/CAM) crowns, cone beam computed tomography (CBCT).
Digital dentistry includes the broad array of technologies that brings the communication, documentation, manufacture and delivery of dental therapy under the umbrella of computer-based algorithms [1].

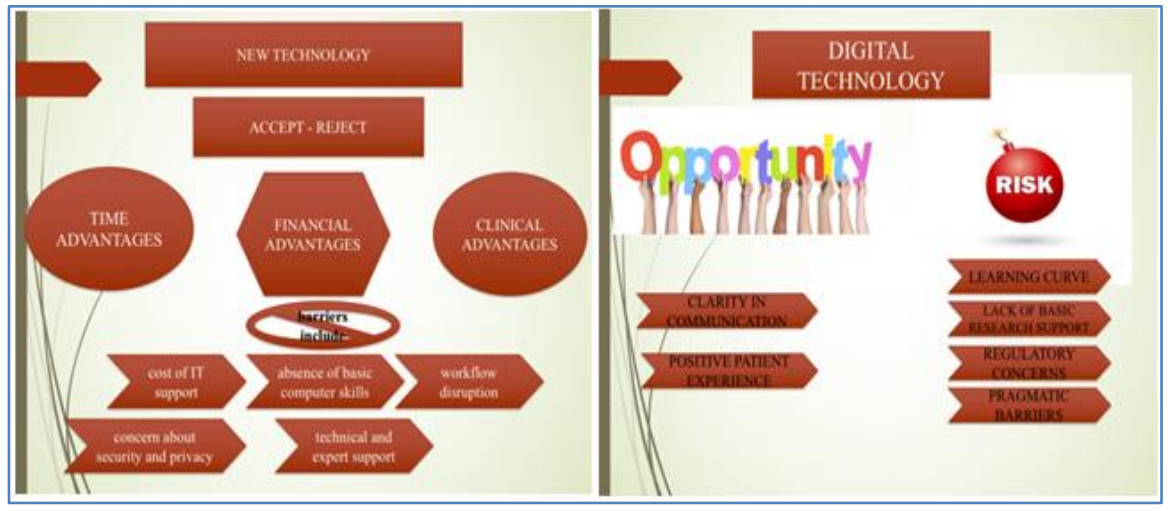

"We as prostodontist treat individualized patients, not computerized machines."

Digital dental technology, a recent survey revealed that approximately $50 \%$ of post-graduate prosthodontic programs included aspects of this technology in their programs while only $12 \%$ of undergraduate programs included this technology in their curriculum.

Faculty determined CAD/ CAM chair-side crowns were equal or better quality than laboratory fabricated PFM or metal crowns. 


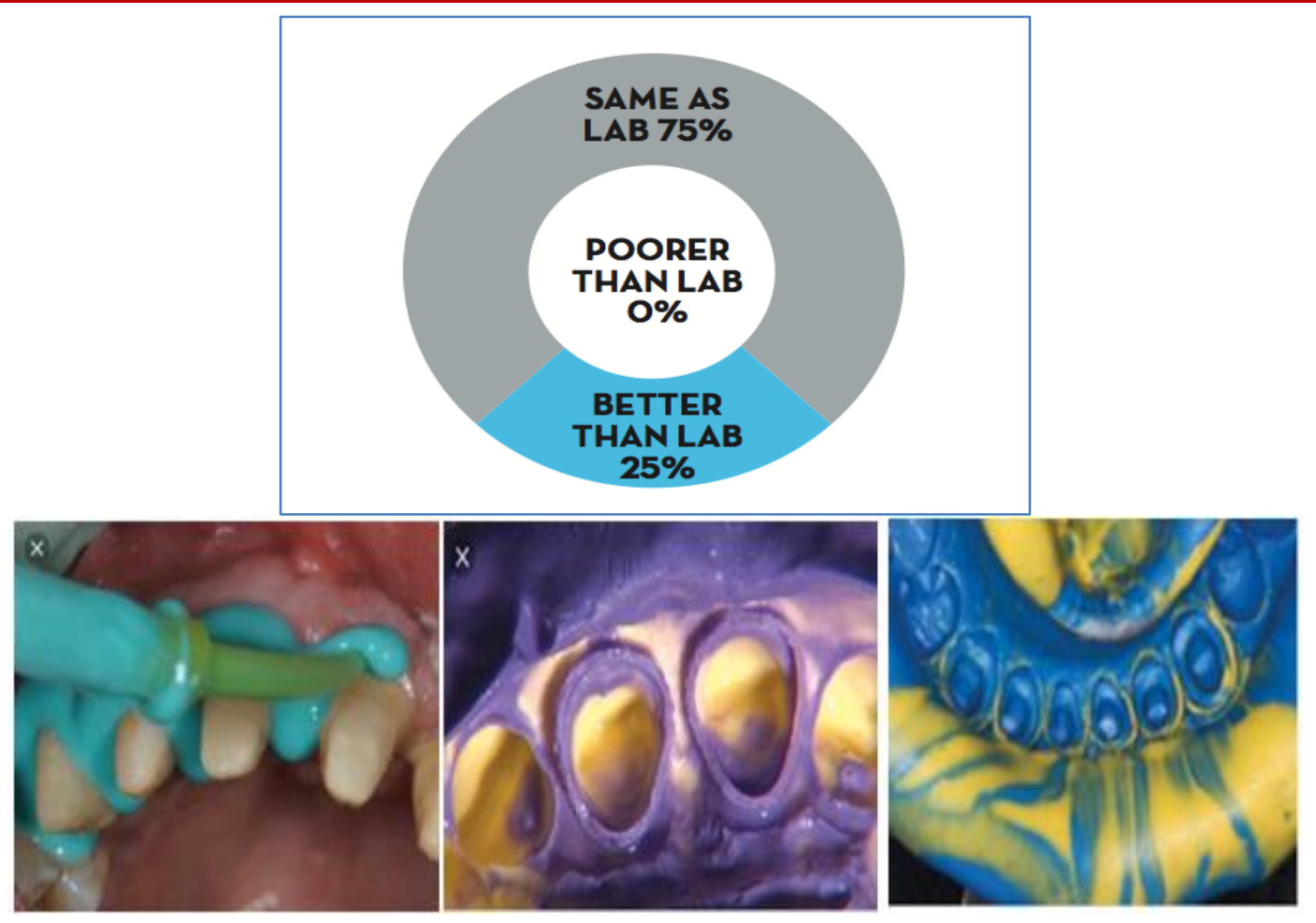

The conventional impression materials used for definitive impressions in fixed prosthodontics that is polyether and poly vinylsiloxane exhibit excellent dimensional stability and precision and have been used in fixed prosthodontics for many years.

Digital versus conventional impressions for fixed prosthodontics: A systematic review and metaanalysis [2].

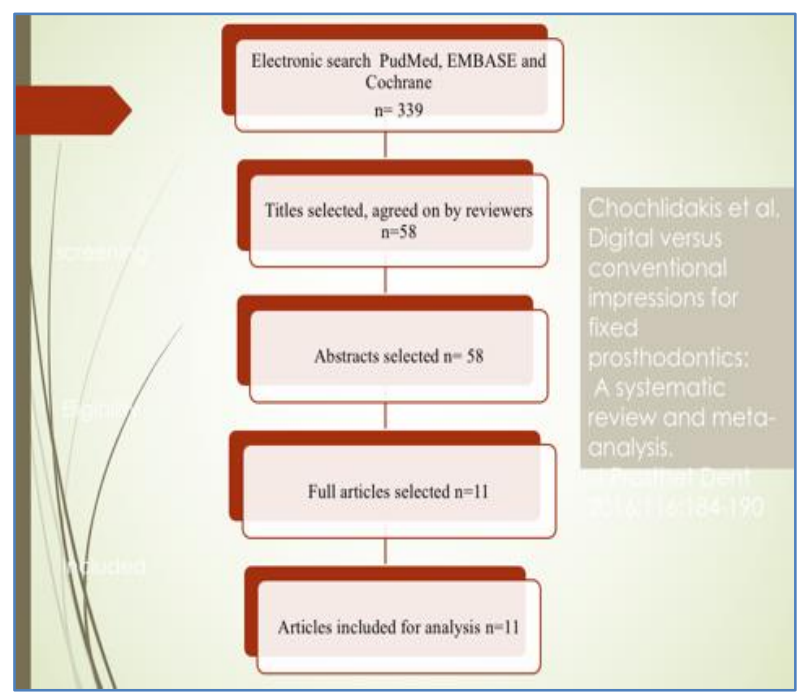

Within the limitations of the systematic review and meta-analysis, the following conclusions were given

1. Dental restorations fabricated with digital impression technique showed similar marginal discrepancies compared with those obtained with the conventional impression technique.
2. Digital dies had restorations with smaller marginal and internal discrepancy compared with SLA/polyurethane dies.

3. Pressing and CAD-CAM fabrication techniques, similar results found for both the marginal and the internal discrepancy in conventional and digital groups.

4. Yuzbasioglu et al. in 2014 compared the two impression techniques in terms of patient preferences and treatment comfort [3].

Digital versus conventional techniques for pattern fabrication of implant- supported frameworks [4]

- No significant differences in retentive values between frameworks cast from conventional and rapid prototyped wax patterns.

- The retentions achieved by all three groups were clinically acceptable.

- Frameworks cast from CAD/CAM waxing preparation required significantly more adjustments compared to conventional or rapid prototyped wax patterns.

Digital impressions compared with Conventional Impressions in Fixed Prosthodontics [5].

- Accuracy of digital impression is at the same level as conventional impression methods for crowns and short FPDs, thus both techniques can be used.

- Fabrication of implant-supported crowns and FPDs, the digital impressions also result in a clinically acceptable fit; however, for large, fullarch FPD conventional impression technique results in better accuracy compared to the digital 
method, which is why the conventional methods may be preferred.

- More number of studies and additional investigations are needed to confirm the findings.

Indications and contraindications of dental implants in medically compromised patients [6]. In order to analyze the indications and contraindications of treating MCP with dental implants. A research was carried out on PubMed using the key words implant, systemic disease or medically compromised), in articles published from 1993 to 2013.

- 64 articles were found, from which 16 met inclusion criteria. The survival rate of dental implants placed in MCP who suffer from controlled systemic diseases does not indicate a total or partial contraindication for the placement of dental implants.

- Oral bisphosphonates by patients who suffer from osteoporosis seems to be a partial contraindication for the treatment with dental implants and the patient must understand the necessity of a longer follow-up period to detect any sign of $\mathrm{BCN}$.

- Radiotherapy protocols in the head or neck region, with doses higher than 50Gy, show lower levels of osseo-integration, being contraindicated their placement in those patients who have received a therapy with bisphosphonates intravenously and when they are associated with hormonal therapy, corticosteroids or immune-supressors.

- The consumption of tobacco seems to be a factor associated with the increase in the loss of dental implants; Wilson and Nunn established a failure rate 2.5 times higher in patients who smoke.

- Conventional prosthetic protocols are advantageous in such situations.

Conventional Implant Site Preparation Method versus Osseodensification[7]

New Osseodensification Implant Site Preparation Method to Increase Bone Density in LowDensity Bone: In Vivo Evaluation in Sheep.

\section{Osseodensification}

- Increase implant primary stability

- Increase the percentage Bone Volume around dental implants inserted in low-density bone.

- Future in vivo human studies are needed to confirm the results showed in the present article.

\section{Comparison of Marginal Bone Loss in One-stage versus Two-stage Implant Surgery [8]}

- Study was conducted to compare the crestal bone loss at distal and mesial surface of implants installed through either one-stage or two- stage surgical approach.

The mean bone loss on the mesial and distal surfaces of implants through one-stage surgery and two-stage surgery was $0.76 \pm 0.04$ and $0.842 \pm 0.04$ mm respectively.

- One-stage surgical technique may provide better esthetic and function for dental implants. There is no significant difference between the two approaches concerning the marginal bone loss.

Replacement of mandibular molars with single-unit restorations supported by wide-body implants: immediate versus delayed loading.-A randomized controlled study [9].

\section{PURPOSE}

Compare single implant-supported mandibular molar restorations using either an immediate or a delayed loading protocol.

\section{RESULTS}

- No implants lost in the delayed loading group $(0 / 15)$ and one implant failed (1/15) in the immediate loading group.

- No differences were observed in relation to implant length or insertion torque between the groups.

- The average radiographic bone level change after 1 year of function was $1.2+/-0.55 \mathrm{~mm}$ (range, 0.5 to $2.6 \mathrm{~mm}$ ) and $0.77+/-0.38 \mathrm{~mm}$ (range, 0.29 to 1.23 $\mathrm{mm}$ ) for the delayed loading and the immediately loading implants, respectively.

\section{Maxillary immediate implant loading [10]}

- The quality and quantity of bone are the two most important factors that affect primary stability.

Maxillary immediate implant placement can be quite challenging because of factors like:

1. Lesser bone density

2. Thin cortical plate

3. Proximity to the maxillary sinus

The observation with immediate implant placement in fresh extraction sockets followed by immediate loading (preferably non-functional) were-

(1) The esthetic outcome was equal to the conventional approach.

(2) Similar survival rates for conventional loading can be achieved at single implant sites when rough surfaced implants, achieving high torque values, are placed by experienced clinicians.

- Disadvantages may include risk of failure due to residual periosteal infection, unpredictable site morphology, a potentially limited amount of soft tissue.

- Taledo et al. placed distal implants in angulated manner, which presented with bone loss similar to conventional implant placement. 


\section{CONCLUSION}

- One of the most critical aspects of high quality patient care in prosthodontics is the technical flow chart followed to accomplish the treatment plan.

- Dentistry has evolved over the year by leaps and bound, from conventional plaster and impression compound to Osseo-densification and navigational implant surgeries and aesthetic make over the face.

- Recent advances in prosthodontics look very appealing, but their major drawback is the fact that they lack substantial supporting literature and data.

- Compare two stage implant surgery to single stage surgery, delayed loading protocol to immediate loading, conventional impressions to digital impressions, and the variation in results is not vast.

- The newer techniques are time saving, but data has been collected from FEA, animal studies (bovine/ porcine) and an extended time frame is required before results in human can be analyzed and documented.

- These advanced procedures look very attractive, but their feasibility of execution needs to be reviewed.

\section{REFERENCES}

1. Lyndon, F., Mark, E. The current impact of digital technology in prosthodontics. ACP.

2. Digital versus conventional impressions for fixed prosthodontics. (2016). A systematic review and meta-analysis Chochlidakis. JPD;116;184-190

3. Yuzbasioglu. (2014). Comparision of digital and conventional impression techniques:evaluation of patients' preception, treatment comfort,effectiveness and clinical outcome, 14(10);1-7.

4. Digital versus conventional techniques for pattern fabrication of implant- supported frameworks
Alikhasi. (2018). European Journal of General Dentistry, 12:71-6.

5. Digital versus Conventional Impressions in Fixed Prosthodontics. (2016). A Review Ahlholm JPACP, 1-7.

6. Indications and contraindications of dental implants in medically compromised patients. (2003). Rafael Gómez-de Diego.Clinical implant dentistryand related research, 5(1)1:57-60.

7. Trisi. (2016). Conventional Implant Site Preparation Method versus Osseodensification. Implant Dentistry, 25(1):24-31.

8. Rafael Gómez-de Diego. (2014). Indications and contraindications of dental implants in medically compromised patients: Updatemed oral patol oral cir buccal, 1(19);483-89.

9. Conventional Implant Site Preparation Method versus Osseodensification Trisi. (2016). Implant dentistry, 25(1): 24-31.

10. Gheisari, R., Hesamuddin, E., Alavian, A. (2017). Comparison of the Marginal Bone Loss in Onestage versus Two-stage Implant Surgery. J Dent Shiraz Univ Med Sci., 18(4): 272-276.

11. Chochlidakis, K. M., Papaspyridakos, P., Geminiani, A., Chen, C. J., Feng, I. J., \& Ercoli, C. (2016). Digital versus conventional impressions for fixed prosthodontics: A systematic review and meta-analysis. The Journal of prosthetic dentistry, 116(2), 184-190.

12. Aparicio, C., Rangert, B., \& Sennerby, L. (2003). Immediate/early loading of dental implants: a report from the Sociedad Espanola de Implantes World Congress consensus meeting in Barcelona, Spain, 2002. Clinical implant dentistry and related research, 5(1), 57-60.

13. Abichandani, S. J., \& Nadiger, R. (2013). Maxillary immediate implant loading: A comprehensive review. Journal of Dental Implants, 3(1), 52. 